Cite as: Jaspal, R., \& Lopes, B. (2021). Discrimination and mental health outcomes in British Black and South Asian people during the COVID-19 outbreak in the UK. Mental Health, Religion and Culture. https://doi.org/10.1080/13674676.2020.1871328

\title{
Discrimination and mental health outcomes in British Black and South Asian people during the COVID-19 outbreak in the UK
}

\author{
Rusi Jaspal \\ Nottingham Trent University, UK \\ rusi.jaspal@cantab.net \\ Barbara Cristina da Silva Lopes \\ University of Coimbra, Portugal
}

\begin{abstract}
This study focuses on the impact of the COVID-19 outbreak on mental health outcomes in Black and South Asian people in the United Kingdom. A sample of 226 participants completed a survey consisting of measures of ethnic identification, religiosity, British national identification, perceived discrimination, fear of COVID-19, generalized anxiety, depression and life satisfaction. Black participants reported more frequent ethnic discrimination than South Asians who, conversely, reported more religious discrimination. Structural equation modelling showed that discrimination had a direct impact on fear of COVID-19 and an indirect impact through decreased British national identification and life satisfaction. Religiosity and ethnic identification appeared to be protective against fear of COVID-19. Fear of COVID-19 was in turn associated with increased depression and generalized anxiety and decreased life satisfaction. The results suggest that some minority groups may be facing poorer mental health outcomes due to discrimination and minority stress.
\end{abstract}

\section{Keywords}

COVID-19, minority stress, social identity, discrimination, depression, anxiety, mental health

\section{Introduction}

In January 2020, the World Health Organization declared the novel coronavirus disease (COVID-19) a Public Health Emergency of International Concern, and on 11 March it was formally designated a global pandemic. In the absence of effective treatments and a vaccine, many governments, including that of the United Kingdom (UK), implemented a policy of social and physical distancing during the initial outbreak (Public Health England, 2020). It is becoming increasingly clear that both the pandemic and the measures introduced to mitigate it are having deleterious consequences for mental health (Lopes, Bortolon \& Jaspal, 2020).

The UK population is diverse, $14 \%$ of which is of Black, Asian and Minority Ethnic (BAME) background (UK Census, 2011). Recent data from the National Office of Statistics demonstrate that people from this population are disproportionately affected by COVID-19 (ONS, 2020). However, it is unclear whether BAME people are also at greater risk of poor mental health due to the outbreak of the disease. Consistent with the social identity perspective in social psychology (Jetten, Reicher, Haslam \& Cruwys, 2020), it is thought that key social group memberships (such as British national identity) are related to better mental health outcomes in the context of the pandemic. According to the minority stress theory (Meyer, 2003), BAME people may be at risk of psychological adversity due to their minority status. However, these hypotheses have not been investigated in BAME people in the context of COVID-19. This is an important empirical question because, in previous research, it has been observed that BAME people face multiple types of discrimination and that they report 
poorer mental health outcomes than the general population (Maxwell, 2009). Accordingly, this crosssectional survey study provides an integrated approach by examining the contribution of key social identity variables to predicting mental health outcomes in Black and South Asian people during the outbreak in the UK.

\section{The impact of COVID-19 on health and wellbeing}

In addition to the evident physical health risks associated with COVID-19, there is growing concern about its relation to psychopathology, such as depression and anxiety (Hyland et al., 2020; Kanter \& Manbeck, 2020; Shevlin et al., 2020; Torales et al., 2020). It is thought that self-isolation associated with social distancing measures introduced in the UK may induce, or aggravate, depression, stress and anxiety in the general population (Kanter \& Manbeck, 2020). According to new research, social psychological variables (such as decreased political trust, lacking a strong social network, and fear of COVID-19) appear to be robust predictors of the onset of poor mental health (Jaspal, Lopes \& Lopes, 2020a, 2020b).

Marginalized groups in society are generally found to be especially vulnerable to poor physical and psychological health outcomes (Patel et al., 2018 for a review). It has now been confirmed that people from BAME groups in the UK are at elevated risk of adversity relating to COVID-19, with Black people being more than four times more likely to die of the disease than White people and with Pakistani men being almost twice as likely to die of it than White men (ONS, 2020). However, the risk of poor mental health related to COVID-19 for people in these groups is unclear and is, thus, the focus of this study.

\section{Social identity in Black, Asian \& Minority Ethnic (BAME) communities}

The impact of significant social group memberships in BAME communities on their mental health is examined. These include ethnicity, religion and British national identity, which are not static but are undoubtedly influenced by social context. Significant societal events, such as the September 11th attacks in New York in 2001, the July $7^{\text {th }}$ bombings in London in 2005 and the Black Lives Matter human rights movement which has highlighted systemic racism toward Black people in the US and elsewhere, have clearly affected intergroup relations and raised questions about national identity and belonging (Cinnirella, 2014; Jaspal, Lopes \& Breakwell, 2020). In an analysis of UK government data, Maxwell (2009) found that South Asians were more likely to self-identify as British than Black Caribbean respondents. However, there is also evidence that some South Asians experience difficulties in reconciling their national and ethnic identities (Sekhon \& Szmigin, 2011), due partly to perceived discrimination from the White British majority (Maxwell, 2006). Crucially, individuals may face discrimination due to their ethnicity and/or religion, with British South Asians being more susceptible to the latter due to increasing Islamophobia (Allen, 2010).

Moreover, ethnic minority individuals, who already face prejudice and discrimination due to their group memberships, may suffer double stigma when also faced with the burden of mental illness (e.g., Gary, 2005). BAME groups are vulnerable to poor mental health due to multiple discrimination in relation to their ethnicity and religion, which has been found to prompt the onset of anxiety and depression disorders (Alvarez-Galvez \& Rojas-Garcia, 2019). Moreover, in the UK, it has been found that ethnic minorities across all generations exhibit lower levels of life satisfaction than the White British majority (Knies, Nandi \& Platt, 2016). Poor mental health may be even more accentuated in the context of COVID-19, where ethnic minorities may be more sensitive to discrimination and fear thereby prompting poor mental health.

According to social identity theory (Tajfel \& Turner, 1986), national identity can be thought of in terms of a social group membership which becomes salient in some contexts and which provides feelings of distinctiveness and self-esteem (Spinner-Halev \& Theiss-Morse, 2003). However, due to perceived discrimination, individuals from BAME communities may perceive a 'racial boundary' to Britishness and, thus, perceive it to be inaccessible to them (Vadher \& Barrett, 2009). In the face of 
perceived discrimination and exclusion from the national ingroup, ethnic minorities may develop a stronger relationship with their ethnicity and religion, which not only buffers the deleterious psychological impact of exclusion but also constitutes an alternative source of belonging and social support (Yoo \& Lee, 2005). Nevertheless, perceived discrimination is psychologically aversive and may impair effective coping in response to major stressful events, such as the COVID-19 pandemic. This may in turn increase fear levels and the risk of poor mental health in response to the pandemic.

All in all, there is evidence that, when considering two major minority ethnic groups in the UK (South Asians and Black people), there may be differences in relation to the type of minority stressor that they experience (e.g., racism, religious discrimination) and its subsequent impact on their mental health. Different types of discrimination (religious vs. ethnic) may affect these groups differently - for instance, British South Asians tend to report more religious discrimination than British Black people who, conversely, report more ethnic discrimination (Allen, 2010; Maxwell, 2009). This in turn may impact negatively on their sense of Britishness, fear of COVID-19, life satisfaction and mental health.

\section{Social identity, minority stress and mental health}

This study draws on tenets of both social identity theory, which describes the psychological process of identity construction on the basis of salient social group memberships, and minority stress theory (Meyer, 2003), which has become a dominant theoretical lens for examining the relationship between experiences of discrimination and mental health outcomes in stigmatized minority groups.

According to social identity theory, social identity refers to the dimension of identity derived from identification with relevant social groups. Meaningful social identities, such as national identity and ethnic identity, can perform positive functions for psychological wellbeing, especially in face of a psychological stressor, such as the COVID-19 outbreak and the negative affect, such as fear, that this can generate. Indeed, Jetten, Reicher, Haslam and Cruwys (2020) have argued for a social identity approach to understanding human responses to COVID-19 and its impact on psychological health.

Social identity can be protective because it promotes a sense of shared experience with other group members (Drury, Brown, Gonzalez \& Miranda, 2016), access to social support (Jetten, Haslam \& Haslam, 2012), and a 'gateway' to other group memberships which can alleviate feelings of loneliness (Wakefield et al., 2019). In short, robust group memberships appear to provide a buffer against psychological stress when one is exposed to a stressor (such as the COVID-19 outbreak) (Haslam et al., 2008). On the one hand, the social distancing measures introduced both in the UK and elsewhere can undermine access to social groups. On the other hand, this may be accentuated for BAME groups, since it is clear that not everyone has access to relevant social identities, such as Britishness, and that one is discriminated against because of meaningful social identities, such as one's ethnicity. This can impede healthy coping in the face of COVID-19.

Minority stress theory posits that individuals from minority groups (including ethnic minority groups) are exposed to various stressors which can undermine mental health. In this study, we focus solely on the stressors of ethnic discrimination and religious discrimination in BAME groups, as possible barriers to Britishness (Maxwell, 2009) and as antecedents to poor mental health (Pieterse, Todd, Neville \& Carter, 2012). Indeed, it has been shown in minority stress theory research that exposure to distal stressors can increase the risk of poor mental health outcomes (Meyer, 2003). Building on this existing work, we hypothesize that the experience of distal stressors can decrease the strength of British national identification, on the one hand, and increase negative affect (e.g. fear of COVID-19) and the incidence of poor mental health (e.g. anxiety, depression), on the other hand.

\section{Protective factors against poor mental health in BAME people in the UK}

The UK is a religiously diverse country and, for many BAME people, religion is an important group membership. Religion can provide social support, a sense of connection and meaning and, crucially, exposure to social norms associated with the group membership (Ysseldyk, Matheson \& Anisman, 
2010). As Pargament (1997) has found, in the face of potentially fatal disease (as COVID-19 has proven to be), people may begin to cope spiritually when religion is available and thus derive respite from the psychological stress associated with it (see Jaspal, Lopes \& Lopes, 2020a). Religion is also strongly associated with both ethnic identification and life satisfaction (Lim, 2016), which are protective factors against poor mental health. Therefore, it is vital to examine whether religiosity in BAME people in the UK may be buffering against poor mental health.

Given that COVID-19 is being framed in the UK as a national issue, which requires a coordinated response from the British people (cutting across ethnic, religious and other social groups), it appears that national identity is an especially important social group membership in this context (e.g. Nerlich \& Jaspal, 2020). Yet, it has been shown that individuals from marginalized and stigmatized communities, such as BAME people, may have a less strong sense of British national identity due to perceived discrimination and exclusion but that they may derive support from their ethnic and religious group identities (Maxwell, 2009; Vadher \& Barrett, 2009). However, it is also true that these group memberships may also be compromised by ethnic and religious discrimination (e.g. racism, Islamophobia).

\section{Hypotheses}

Drawing on social identity theory and minority stress theory, the following hypotheses are tested:

1. There should be statistically significant differences between ethnic groups (British South Asians vs. Black British people) for strength of ethnic identification, strength of British national identification, fear of COVID-19, and generalized anxiety.

2a. Discrimination, fear of COVID-19, group identification and life satisfaction should predict the variance of depression, with discrimination and fear of COVID-19 being positively related to depression, and group identification and life satisfaction being inversely related to it.

2b. Discrimination, fear of COVID-19, ethnic group membership, and life satisfaction should predict the variance of generalized anxiety, with British South Asians being more susceptible to anxiety, and discrimination and fear of COVID-19 being positively related to anxiety. Moreover, life satisfaction should be inversely associated with anxiety.

3. In light of the literature highlighting ethnic group differences (British South Asians vs. British Black) in type of discrimination they face and their strength of British national identification, it is expected that the relationships between ethnic groups and the mental health variables of depression and generalized anxiety should be mediated by the social identity variables (religiosity, strength of ethnic and British national identification, and different types of discrimination: religious and ethnic discrimination), fear of COVID-19 and life satisfaction.

\section{Ethics}

\section{Methods}

Nottingham Trent University's College of Business, Law and Social Sciences Ethics Committee provided ethical clearance for this research project (REF 2020/118).

\section{Participants}

A sample of 226 BAME people was recruited on Prolific ${ }^{1}$ and completed a survey concerning social identity, COVID-19 and mental health in May 2020. There were 81 Indians (35.8\%), 40 Pakistanis (17.7\%), 48 Black Africans (21.2\%) and 57 Black Caribbeans (25.3\%). The majority of the participants self-identified as Christians $(N=86,38.1 \%)$, followed by Muslims $(N=50,22.1 \%)$, no religion $(N=39,17.3 \%)$, Hindu $(N=36,15.9 \%)$, Sikh $(N=13,5.8 \%)$ and Jain $(N=2,0.9 \%)$. There was

\footnotetext{
${ }^{1}$ Prolific is an online recruitment platform for surveys. Users with access to the Internet who meet the eligibility criteria sign up to participate in the survey and are paid a rate set by the researcher to complete the survey. https://www.prolific.co/
} 
a fairly even distribution of males and females - 124 (54.9\%) and 102 (45.1\%), respectively. The mean age was $29.8(S D=8.70)$, with a range of $18-69$. The majority reported having an undergraduate degree $(N=111,49.1 \%)$; 56 A-levels $(24.8 \%) ; 48(21.2 \%)$ a postgraduate qualification; and $11(4.9 \%)$ as having a GCSE qualification.

\section{Measures}

\section{Strength of ethnic identification}

The 6-item Multigroup Ethnic Identity Measure-Revised (Phinney \& Ong, 2007) was used to measure strength of ethnic identification. Sample item: 'I have a strong sense of belonging to my own ethnic group'. Participants indicated the extent to which they agreed or disagreed with each statement ( $1=$ strongly disagree to $5=$ strongly agree). Possible scores ranged from 6 to 42 , and a higher score indicated stronger ethnic identification $(\alpha=.87)$.

\section{Religiosity}

The 5-item Brief Version of the Santa Clara Strength of Religious Faith Questionnaire (Plante et al., 2002) was used to measure religiosity. Sample item: 'I pray daily'. Participants indicated the extent to which they agreed or disagreed with each statement (1=strongly disagree to $4=$ strongly agree). Possible scores ranged from 5 to 20 , and a higher score indicated higher religiosity $(\alpha=.94)$.

\section{Strength of British national identification}

The 7-item British National Identity Scale (Cinnirella, 1997) was used to measure strength of British national identification. Sample item: 'To what extent do you feel British?' Participants indicated responses on a Likert scale ( $1=$ not at all to $5=$ extremely). Possible scores ranged from 7 to 35 , and a higher score indicated stronger British national identification $(\alpha=.85)$.

\section{Perceived ethnic discrimination}

The 9-item Everyday Discrimination Scale (Williams, Jackson, Yu \& Anderson, 1997) was used to measure the frequency of discriminatory events. Participants were asked to think about the events occurring because of their ethnicity. Sample item: 'People act as if they're better than you are'. Participants indicated the frequency of these events ( $1=$ never to $6=$ almost every day). Possible scores ranged from 9 to 54, and a higher score indicated higher levels of perceived discrimination due to one's ethnicity $(\alpha=.90)$.

\section{Perceived religious discrimination}

The same 9-item Everyday Discrimination Scale was used to measure the frequency of discriminatory events and participants were asked to think about the events occurring because of their religion. A higher score indicated higher levels of perceived discrimination due to one's religion $(\alpha=.94)$.

\section{Fear of COVID-19}

The 10-item Fear of COVID-19 Scale (Ahorsu et al., 2020) was used to measure fear levels due to COVID-19. Sample item: 'I am most afraid of COVID-19'. Participants indicated the extent to which they agreed or disagreed with each statement $(1=$ strongly disagree to $5=$ strongly agree). Possible scores ranged from 10 to 50, and a higher score indicated higher fear of COVID-19 $(\alpha=.90)$.

\section{Generalized anxiety}

The 7-item Generalized Anxiety Disorder Assessment (Spitzer et al., 2006) was used to measure symptoms of generalized anxiety over the last two weeks. Sample item: 'Not being able to stop or control worrying?' Participants indicated the frequency of these experiences ( $0=$ not at all to $3=$ nearly every day). Possible scores ranged from 0 to 21 . A higher score indicated higher generalized anxiety 
and the authors suggest that scores of $\geq 5, \geq 10$ and $\geq 15$ indicate mild, moderate and severe anxiety, respectively $(\alpha=.92)$.

\section{Depression}

The 10-item Center for Epidemiologic Studies Depression Scale Revised (Radloff, 1977) was used to measure depression. Sample item: 'I felt that everything I did was an effort'. Participants indicated the frequency of these perceptions during the last week ( $0=$ rarely or none of the time [less than 1 day] to $3=$ all of the time [5-7 days]). Possible scores ranged from 0 to 30. A higher score indicated higher depression. The authors of the scale suggest that a score of $\geq 10$ indicates mild depression, $\geq 16$ moderate depression and $\geq 21$ severe depression $(\alpha=.70)$.

\section{Life satisfaction}

The 5-item Satisfaction with Life Scale (Diener et al., 1985) was used to measure judgements of life satisfaction. Sample item: 'In many ways my life is close to my ideal'. Participants indicated the extent to which they agreed or disagreed with each statement (1=strongly disagree to $7=$ strongly agree). Possible scores ranged from 5 to 35, and a higher score indicated greater life satisfaction $(\alpha=.88)$.

\section{Normal distribution checks}

\section{Results}

Kolmogorov-Smirnov tests showed that the following variables were not normally distributed: religious discrimination; strength of ethnic identification; life satisfaction; generalized anxiety; and depression. Logarithmic and Square Root Transformations were applied to those variables, resulting in normal distributions.

\section{Descriptive statistics}

The mean for strength of ethnic identification was 31.39 (Median=33, $S D=6.81$ ), suggesting strong ethnic identification in this sample. The mean for religiosity in this sample was $12.97(S D=4.53)$, suggesting moderate religiosity. The means for ethnic and religious discrimination were 2.32 (Median=2.33, $S D=0.81$ ) and 1.58 (Median=1.22, $S D=0.77$ ), respectively, suggesting that discrimination was experienced relatively infrequently but that ethnic discrimination was more common than religious discrimination. The mean for strength of British national identification was $21.84(S D=5.24)$, suggesting a fairly strong sense of British national identity.

The mean score for life satisfaction was 19.39 (Median=20, $S D=6.52$ ), suggesting moderate to high life satisfaction. Fear of COVID-19 presented a $M=25.83, S D=7.96$, suggesting moderate fear of COVID-19. The mean for depression was 10.25 (Median=10, $S D=4.46$ ) and for generalized anxiety it was 5.52 (Median=4.50, $S D=4.94$ ), suggesting moderate depression and generalized anxiety in this sample. There were $122(53.98 \%)$ participants who scored $\geq 10$ suggesting mild depression; 23 who scored $\geq 16$ indicating moderate depression $(10.18 \%)$ and 5 who scored $\geq 21$ suggesting severe depression $(2.21 \%)$. Moreover, there were 68 participants with mild generalized anxiety (30.09\%); 32 with moderate generalized anxiety (14.16\%) and 13 participants with severe generalized anxiety $(5.75 \%)$.

\section{Ethnic differences for the key variables of interest}

A dummy variable was computed to examine ethnic differences between Indians and Pakistanis $=0$ $(N=121)$ vs. Black Africans and Black Caribbeans $=1(N=105)$. Benjamini and Hochberg's (1995) adjusted significance value $q=0.03$ was used to avoid a Type I error. Independent samples $t$-tests bootstrapped at 1000 samples showed statistically significant differences between the two ethnic groups for fear of COVID-19 $[t(222)=2.084, p=0.04]$ and generalized anxiety $[t(222)=2.792$, $p=0.006]$; but not for life satisfaction $[t(222)=-1.411, p=0.16]$ or depression $[t(222)=0.540, p=0.59]$. 
There were also statistically significant differences between the ethnic groups for both ethnic discrimination $[t(222)=-3.567, p<0.001]$ and religious discrimination $[t(222)=3.474, p=0.001]$ and both for strength of British national identification $[t(222)=4.227, p<0.001]$ and strength of ethnic identification $[t(222)=2.448, p=0.02]$.

Indian and Pakistanis reported much more generalized anxiety and fear of COVID-19 than Black Africans and Caribbeans. In contrast, Black Africans and Caribbeans reported much more ethnic discrimination than Indians and Pakistanis, whereas Indians and Pakistanis reported much more religious discrimination than Black Africans and Caribbeans. Also, whereas Indians and Pakistanis exhibited stronger British national identification compared to Black Africans and Caribbeans, Black Africans and Caribbeans exhibited much stronger ethnic identification than Indians and Pakistanis. These results support hypothesis 1. For the means, standard deviations, effect sizes and $95 \%$ CI, see Table 1.

**Insert Table $1 * *$

\section{Stepwise regressions predicting mental health outcomes Depression}

A stepwise regression was conducted with a bootstrap at 1000 samples with blocks of variables (ethnic group $[0=$ British South Asians vs. $1=$ British Black people]; ethnic and religious discrimination; strength of ethnic identification; strength of British national identification; religiosity; fear of COVID-19 and life satisfaction predicting the variance of depression in the context of COVID19 . The model was statistically significant with a $R^{2}=0.23 ; F(4,225)=16.63, p<0.001$. Benjamini and Hochberg's (1995) adjusted significance value was used $q=0.03$.

Fear of COVID-19 emerged as the strongest predictor of the variance of depression with a $\beta=0.30$; S.E. $=0.034 ; t=4.99, p<0.001 ; 95 \%$ CIs $(0.101,0.237)$; followed by life satisfaction with a $\beta=-0.21$; S.E. $=0.036 ; t=-3.43, p=0.001 ; 95 \%$ CIs $(-0.223,-0.0085)$; ethnic discrimination with a $\beta=0.17$; S.E. $=0.372 ; t=2.81, p=0.005 ; 95 \%$ CIs $(0.403,1.801)$; and religiosity with a $\beta=-0.14$; S.E. $=0.050 ; t=-2.27, p=0.02 ; 95 \%$ CIs $(-0.271,-0.065)$. This suggested that ethnic discrimination and fear of COVID-19 are positively associated with depression, while religiosity and life satisfaction are negatively associated with depression.

\section{Generalized anxiety}

A stepwise regression was conducted with a bootstrap at 1000 samples with blocks of variables (ethnic group $[0=$ British South Asians vs $1=$ British Black people]; ethnic and religious discrimination; strength of ethnic identification; strength of British national identification; religiosity; fear of COVID-19 and life satisfaction) predicting the variance of generalized anxiety in the context of COVID-19. The model was statistically significant with a $R^{2}=0.40 ; F(4,225)=36.13, p<0.001$.

Fear of COVID-19 emerged as the strongest predictor of the variance of generalized anxiety with a $\beta=0.43$; S.E. $=0.036 ; t=7.76, p<0.001 ; 95 \%$ CIs $(0.185,0.332)$; followed by life satisfaction with a $\beta=-0.30 ;$ S.E. $=0.43 ; t=-5.69, p<0.001 ; 95 \%$ CIs $(-0.309,-0.133)$; ethnic group with a $\beta=-0.18$; S.E. $=0.398 ; t=-3.31, p=0.001 ; 95 \%$ CIs $(-2.898,-1.007)$; and ethnic discrimination with a $\beta=0.18$; S.E. $=0.374 ; t=3.27, p=0.001 ; 95 \%$ CIs $(0.457,1.801)$. This suggested that, as with depression, ethnic discrimination, fear of COVID-19 are positively associated with generalized anxiety, and life satisfaction negatively associated with generalized anxiety. It was found that being British South Asian is also associated with more generalized anxiety.

These results support hypothesis $2 \mathrm{a}$ and $2 \mathrm{~b}$. For a summary of both multiple stepwise regression models for depression and generalized anxiety, see Tables 2 and 3, respectively.

*Insert Tables 2 and 3 here* 


\section{Structural equation model}

A structural equation model was performed with a bootstrap at 200 with the main predictors (ethnic group [dummy coded: $0=$ British South Asians vs. 1=Black British people]; and of religiosity); the mediators (ethnic discrimination; religious discrimination; strength of ethnic identification; strength of British national identification; fear of COVID-19; life satisfaction) to predict the variance of the mental health variables (depression and generalized anxiety). Model fit was acceptable with a Root Mean Square Error of Approximation (RSMEA) of 0.06, a Tucker-Lewis Index (TLI) $=0.95$ and a Confirmatory Factor Index $(\mathrm{CFI})=0.80$. See Figure 1.

First, ethnic group had a statistically significant direct effect on generalized anxiety with a $\beta=-0.11$, S.E. $=0.514 ; p=0.04$, but there were also statistically significant mediation effects. Ethnic group predicted the variance of both ethnic and religious discrimination $[\beta=0.22$, S.E. $=0.105$; $p<0.001 ; \beta=-0.21$, S.E. $=0.100 ; p=0.002$, respectively]. Then, religious discrimination predicted the variance of generalized anxiety with a $\beta=0.16$, S.E. $=0.333 ; p=0.004$.

In contrast, being Black British was associated with more ethnic discrimination $[\beta=0.22$, S.E. $=0.105 ; p<0.001]$, which then significantly predicted the variance of strength of British national identification with a $\beta=-0.17$, S.E. $=0.419 ; p=0.008$ and fear of COVID-19 with a $\beta=0.26$, S.E. $=0.714$; $p<0.001$. Fear of COVID-19 then significantly predicted the variance of life satisfaction with a $\beta=-$ 0.39 , S.E. $=0.123 ; p=0.010$ and of both depression and generalized anxiety $[\beta=0.31$, S.E. $=0.032$; $p<0.001 ; \beta=0.44$, S.E. $=0.032 ; p<0.001$, respectively]. Moreover, strength of British national identification predicted the variance of life satisfaction with a $\beta=0.17$, S.E. $=0.080 ; p=0.008$ and life satisfaction in turn predicted the variance of fear of COVID-19 with a $\beta=-0.35$, S.E. $=0.205, p=0.04$ and of both depression and generalized anxiety $[\beta=-0.24$, S.E. $=0.039 ; p<0.001 ; \beta=-0.31$, S.E. $=0.039$; $p<0.001$, respectively].

Religiosity impacted on the variance of both depression and generalized anxiety through life satisfaction and strength of ethnic identification. Indeed, religiosity had a statistically significant impact on the variance of strength of ethnic identification with a $\beta=0.26$, S.E. $=0.097 ; p<0.001$, and on the variance of life satisfaction with a $\beta=0.22$, S.E. $=0.097 ; p<0.001$. Strength of ethnic identification also impacted on the variance of life satisfaction with a $\beta=0.25$, S.E. $=0.065 ; p<0.001$. Life satisfaction then impacted on the variance of both depression and generalized anxiety $[\beta=-0.24$, S.E. $=0.039 ; p<0.001 ; \beta=-0.31$, S.E. $=0.039 ; p<0.001]$. These results support hypothesis 3 .

\section{**Insert Figure 1 here**}

\section{Discussion}

This study provides preliminary evidence of the additional mental health burden faced by BAME individuals and shows the pathways through which BAME people in the UK may be at risk of poor mental health outcomes in the context of COVID-19. Though not a prevalence study, our study suggests that the prevalence of all forms of depression $(N=150,66.4 \%)$ and anxiety $(N=113,50 \%)$ is higher than that reported for the general population in eight countries during the pandemic (Xiong et al., 2020) and similar to the prevalence of depressive symptomatology in China where the first outbreak of COVID-19 occurred (Wang et al., 2020). Consistent with social identity theory and minority stress theory, individuals appear to be at greater risk of poor mental health when they face stigma associated with valued identities, such as their ethnicity and religion, and when they are excluded from the national ingroup (Gary, 2005). Moreover, the study provides evidence of a possible buffering effect of social group identification on poor mental health outcomes associated with COVID-19. Yet, these group memberships (i.e. Britishness) may be less available to those who face discrimination (Jaspal, Lopes \& Breakwell, 2020). 
The study reveals clear ethnic differences on key variables, suggesting that particular BAME groups may be more or less at risk of poor mental health. South Asians reported higher levels of fear of COVID-19 and anxiety than Black participants, who, conversely reported more frequent discrimination due to their ethnicity. This is broadly consistent with previous research which shows that British Black people report higher levels of racism in various contexts, which in turn leads them to derive a weaker attachment to Britishness (Maxwell, 2009). Conversely, South Asians reported higher religiosity and more frequent discrimination due to their religion compared to Black participants. This could be attributed to growing Islamophobia which directly affects (mainly Pakistani) Muslims but may also affect non-Muslim South Asians who may be miscategorized as Muslim (see Allen, 2010; Jaspal, 2013). It is in this context that the direct impact of ethnic group (i.e. being South Asian) on generalized anxiety should be understood.

Ethnic discrimination does appear to constitute a key variable in our study of mental health amid the COVID-19 pandemic. As suggested in previous research, ethnic discrimination directly impacts on strength of British national identification (Maxwell, 2009). Weak British national identity is in turn associated with decreased life satisfaction, paving the way for increased fear of COVID-19 - this is particularly the case for Black participants. Crucially, ethnic discrimination also has a direct impact on fear of COVID-19. It is possible that frequent experiences of discrimination due to one's ethnicity put BAME groups in a position where they become more vulnerable to fear of COVID-19. Consistent with minority stress theory, discrimination may deprive individuals of feelings of social support associated with belonging, acceptance and inclusion. Furthermore, this may exclude individuals from the collective activities aimed to facilitate inclusion and to boast morale, such as 'Clap for our Carers', ${ }^{2}$ which might normally facilitate effective coping (Jaspal \& Nerlich, 2020). More generally, discrimination is aversive for psychological wellbeing, which in turn may be aggravated by the arrival of a significant stressor, namely the outbreak of a life-changing, and potentially life-limiting, disease for which there is currently no available vaccine.

Fear is an important construct to consider during the pandemic (Breakwell \& Jaspal, 2020). It is an emotional and physiological reaction to a hazard (in this case, infection with COVID-19) and can be psychologically distressing (Shultz et al., 2016). Fear is not unique to COVID-19 but has been observed in response to various disease outbreaks, including Ebola and HIV/AIDS (Ahorsu et al., 2020; Towers et al., 2015). However, as demonstrated in this study, it does have clear pathways to decreased life satisfaction and to increased depression and generalized anxiety. Those who face discrimination and have decreased life satisfaction may be at greater risk of this adverse emotional reaction to the pandemic. This in turn may put them at increased risk of poor mental health. It is worth noting that fear and perceived risk of COVID-19 are conceptually distinct constructs and that decreased fear does not necessarily amount to decreased perceived personal risk (Jaspal, Fino \& Breakwell, 2020).

Our study also provides preliminary evidence of the protective role of identification with valued ingroups, which is a key tenet of social identity theory. On the one hand, British national identification is associated with life satisfaction, which in turn has a negative impact on fear of COVID-19. However, this identity is not unanimously endorsed by all BAME people, and especially those of Black ethnicity, many of whom may perceive it to be inaccessible to them due to racism. This finding provides additional evidence of the insidious effects of stigma and exclusion from the national ingroup and highlights the need to re-fashion Britishness so that it can become accessible to all Britons, regardless of ethnicity and religion. Furthermore, religiosity has two specific benefits: on the one hand, it enhances life satisfaction (Okulicz-Kozaryn, 2010), which in turn impacts on fear of COVID-19 and, on the other hand, it provides a 'gateway' to identification with one's ethnic group,

\footnotetext{
2 'Clap for Carers' was an initiative in the UK to show support for key workers (such as doctors, nurses, healthcare workers and others), to facilitate a sense of inclusion and to boast morale in the face of COVID-19
} 
which has the same favorable effect on life satisfaction (see Lim, 2016). This again provides support for the hypothesis that identification with valued social groups enhances wellbeing. Crucially, it is life satisfaction that perhaps enables BAME individuals to put matters into perspective, to manage their COVID-19 fear levels and, thus, to experience better mental health outcomes.

\section{Limitations}

First, this study focuses on a limited number of BAME groups but there are significant groups in the UK (such as Bangladeshis and Latin Americans) which should be studied in future research. Second, although there is a strong indication that decreased group identification is a pathway towards poor mental health due to decreased social support (McGuire \& Miranda, 2008), social support was not explicitly measured in this study. Further research should consider this as a mediator. Third, while this cross-sectional study provides preliminary evidence concerning risk of poor mental health, the hypotheses in this study should be tested using an experimental design, which would enable us to ascertain the causal effect of discriminatory experiences on fear of COVID-19 and the mental health variables.

\section{Conclusions}

This study shows that different types of perceived discrimination may put BAME groups at increased risk of poor mental health during the COVID-19 pandemic. Moreover, identification with social groups that are generally valued by BAME groups, such as ethnicity and religion, and with the national group may enhance life satisfaction, thereby promoting more effective and sustainable psychological responses to the pandemic. It is important to acknowledge that some groups may be facing poorer outcomes due to the social and psychological barriers to accessing robust support. To tackle the mental health burden of COVID-19, existing resources will be valuable, including the digitized Cognitive and Behavioural Therapy (CBT) at Step 2 in the National Health Service (NHS) in the UK, which could be adapted to the specific stressors faced by BAME people during the pandemic. This tailored digital CBT could include components of mindfulness, be provided in a socially distanced fashion, and have clinical psychologists and/or CBT therapists on call to address emerging clinical issues. Indeed, CBT and mindfulness-based approaches have been shown to be effective in treating depressive symptomatology (Arch \& Ayers, 2013). Specifically, the digital CBT ought to include components of stress and fear management and the development of coping skills (e.g., how to cope with discrimination) (Kompus, 2018). More generally, a recurrent theme in many previous disease outbreaks - HIV, Ebola and Zika - is that of the insidious effect of stigma on the health and wellbeing of minority groups. In order to alleviate the burden of poor mental health during and after the pandemic, stigma due to one's valued group memberships must continue to be challenged.

\section{Disclosure of potential conflicts of interest}

The authors declare that they have no conflict of interest.

\section{Data sharing statement}

The dataset can be accessed by e-mailing the corresponding author.

\section{References}

Ahorsu, D. K., Lin, C. Y., Imani, V., Saffari, M., Griffiths, M. D., \& Pakpour, A. H. (2020). The Fear of COVID-19 Scale: Development and initial validation. International Journal of Mental Health and Addiction. https://doi.org/10.1007/s11469-020-00270-8

Allen, C. (2010). Islamophobia. London: Ashgate.

Alvarez-Galvez, J. \& Rojas-Garcia, A. (2019) Measuring the impact of multiple discrimination on depression in Europe. BMC Public Health, 19(435). https://doi.org/10.1186/s12889-019-6714-4 
Arch, J. J., \& Ayers, C. R. (2013). Which treatment worked better for whom? Moderators of group cognitive behavioral therapy versus adapted mindfulness based stress reduction for anxiety disorders. Behaviour Research and Therapy, 51(8), 434-442. https://doi.org/10.1016/j.brat.2013.04.004

Benjamini, Y. \& Hochberg, Y. (1995) Controlling the false discovery rate: a practical and powerful approach to multiple testing. Journal of the Royal Statistics Society Series B, 57, 289-300. https://doi.org/10.1111/j.2517-6161.1995.tb02031.x

Breakwell, G. M., \& Jaspal, R. (2020). Identity change, uncertainty and mistrust in relation to fear and risk of COVID-19. Journal of Risk Research. https://doi.org/10.1080/13669877.2020.1864011

Cinnirella, M. (1997). Towards a European identity? Interactions between the national and European social identities manifested by university students in Britain and Italy. British Journal of Social Psychology, 36(1), 19-31. https://doi.org/10.1111/j.2044-8309.1997.tb01116.x

Cinnirella, M. (2014). Understanding Islamophobic prejudice: The interface between Identity Process Theory and Intergroup Threat Theory. In R. Jaspal \& G. M. Breakwell (eds.), Identity Process Theory: Identity, Social Action and Social Change (pp. 253-269). Cambridge: Cambridge University Press.

Diener, E., Emmons, R. A., Larsen, R. J., \& Griffin, S. (1985). The Satisfaction with Life Scale. Journal of Personality Assessment, 49(1), 71-75. https://doi.org/10.1207/s15327752jpa4901 13

Drury, J., Brown, R., González, R., and Miranda, D. (2016) Emergent social identity and observing social support predict social support provided by survivors in a disaster: Solidarity in the 2010 Chile earthquake. European Journal of Social Psychology, 46(2), 209- 223. https://doi.org/10.1002/ejsp.2146.

Gary, F. A. (2005) Stigma: Barrier to mental health care in ethnic minorities. Issues in Mental Health Nursing, 26(10), 979-99. https://doi.org/10.1080/01612840500280638

Haslam, C., Holme, A., Haslam, S. A., Iyer, A., Jetten, J., \& Williams, W.H. (2008). Maintaining group memberships: Social identity continuity predicts well-being after stroke. Neuropsychological Rehabilitation, 18(5-6), 671-691. https://doi.org/10.1080/09602010701643449

Hyland, P., Shevlin, M., McBride, O., Murphy, J., Karatzias, T., Bentall, R., Martinez, A. \& Vallières, F. (2020) Anxiety and depression in the Republic of Ireland during the COVID-19 pandemic. Acta Psychiatrica Scandinavica, 142(3), 249-256. https://doi.org/10.1111/acps.13219

Jaspal, R. (2013). British Sikh identity and the struggle for distinctiveness and continuity. Journal of Community and Applied Social Psychology, 23(3), 225-239. https://doi.org/10.1002/casp.2115

Jaspal, R., Fino, E., \& Breakwell, G. M. (2020). The COVID-19 Own Risk Appraisal Scale (CORAS): Development and validation in two samples from the United Kingdom. Journal of Health Psychology. https://doi.org/10.1177/1359105320967429

Jaspal, R., Lopes, B., \& Breakwell, G. M. (2020) British national identity and life satisfaction in ethnic minority groups in the United Kingdom. National Identities. https://doi.org/10.1080/14608944.2020.1822793

Jaspal, R., Lopes, B. \& Lopes, P. (2020a) Fear, social isolation in response to COVID-19 in a religiously diverse UK sample. Mental Health, Religion and Culture, 23(5), 427-442. https://doi.org/10.1080/13674676.2020.1784119

Jaspal, R., Lopes, B., \& Lopes, P. (2020b). Predicting social distancing and compulsive buying behaviours in response to COVID-19 in a United Kingdom sample. Cogent Psychology, 7(1), 1800924. https://doi.org/10.1080/23311908.2020.1800924

Jaspal, R. \& Nerlich, B. (2020). Social representations, identity threat and coping amid COVID-19. Psychological Trauma: Theory, Research, Practice and Policy, 2(S1), S249-S251. https://doi.org/10.1037/tra0000773

Jetten, J., Haslam, C., \& Haslam, S. A. (Eds.) (2012). The Social Cure: Identity, Health and WellBeing. Hove: Psychology Press. https://doi.org/10.4324/9780203813195

Jetten, J., Reicher, S., Haslam, S. A., \& Cruwys, T. (2020). Together Apart: The Psychology of COVID-19. London: Sage. 
Kanter, J. \& Manbeck, C. (2020) Covid-19 could lead to an epidemic of clinical depression, and the health care system is not ready for that either. The Conversation, 12th April 2020. https://theconversation.com/covid-19-could-lead-to-an-epidemic-of-clinical-depression-and-thehealth-care-system-isnt-ready-for-that-either-134528

Knies, G., Nandi, A., \& Platt, L. (2016). Life satisfaction, ethnicity and neighbourhoods: Is there an effect of neighbourhood ethnic composition on life satisfaction? Social Science Research, 60, 110124. https://doi.org/10.1016/j.ssresearch.2016.01.010

Kompus, K. (2018) Virtual Reality CBT helps reduce paranoia, anxiety in psychotic disorders. Lancet Psychiatry, 5(3), 189-191. https://doi.org/10.1016/S2215-0366(18)30063-4.

Lim, C. (2016). Religion, time use and affective wellbeing. Sociological Science, 3, 685-709. https://doi.org/10.15195/v3.a29

Lopes, B. Bortolon, C., \& Jaspal, R. (2020). Paranoia, hallucinations and compulsive buying during the early phase of the COVID-19 outbreak in the United Kingdom: A preliminary experimental study. Psychiatry Research. https://doi.org/10.1016/j.psychres.2020.113455

Maxwell, R. (2006). Muslims, South Asians and the British mainstream: A national identity crisis? West European Politics, 29(4), 736-756. https://doi.org/10.1080/01402380600842312

Maxwell, R. (2009). Caribbean and South Asian identification with British society: The importance of perceived discrimination. Ethnic and Racial Studies, 32(8), 1449-1469. https://doi.org/10.1080/01419870802604024

Meyer, I. H. (2003). Prejudice, social stress, and mental health in lesbian, gay, and bisexual populations: Conceptual issues and research evidence. Psychological Bulletin, 129(5), 674-697. https://doi.org/10.1037/0033-2909.129.5.674

McGuire, T. G., \& Miranda J. (2008). New evidence regarding racial and ethnic disparities in mental health: policy implications. Health Affairs, 27(2), 393-403. https://doi.org/10.1377/hlthaff.27.2.393

Nerlich, B., \& Jaspal, R. (2020). Social representations of 'social distancing' in response to COVID19 in the United Kingdom media. Current Sociology.

Okulicz-Kozaryn, A. (2010). Religiosity and life satisfaction across nations. Mental Health, Religion \& Culture, 13(2), 155-169. https://doi.org/10.1080/13674670903273801

ONS (2020). Coronavirus (COVID-19) related deaths by ethnic group, England and Wales: 2 March $2020 \quad$ to $\quad 10 \quad$ April 2020.

https://www.ons.gov.uk/peoplepopulationandcommunity/birthsdeathsandmarriages/deaths/articles/c oronavirusrelateddeathsbyethnicgroupenglandandwales/2march2020to10april2020

Pargament, K. I. (1997). The Psychology of Religion and Coping: Theory, Research, and Practice. New York: Guilford.

Patel, V., Burns, J., Dhingra, M., Tarver, L., Kohrt, B., \& Lund, C. (2018) Income inequality and depression: a systematic review and meta-analysis of the association and a scoping review of mechanisms. World Psychiatry, 17(1), 76-89. https://doi.org/10.1002/wps.20492

Phinney, J. S., \& Ong, A. D. (2007). Conceptualization and measurement of ethnic identity: Current status and future directions. Journal of Counseling Psychology, 54(3), 271-281. https://doi.org/10.1037/0022-0167.54.3.271

Pieterse, A. L., Todd, N. R., Neville, H. A., \& Carter, R. T. (2012). Perceived racism and mental health among Black American adults: A meta-analytic review. Journal of Counseling Psychology, 59(1), 1-9. https://doi.org/10.1037/a0026208

Plante, T. G., Vallaeys, C. L., Sherman, A. C. \& Wallston, K. A. (2002). The development of a brief version of the Santa Clara Strength of Religious Faith Questionnaire. Pastoral Psychology, 50, 359368. https://doi.org/10.1023/A:1014413720710

Public Health England (2020). Guidance on social distancing for everyone in the UK. https://www.gov.uk/government/publications/covid-19-guidance-on-social-distancing-and-forvulnerable-people/guidance-on-social-distancing-for-everyone-in-the-uk-and-protecting-olderpeople-and-vulnerable-adults 
Radloff, L. S. (1977). The CES-D Scale: A self-report depression scale for research in the general population. Applied Psychological Measurement, $1(3), \quad 385-401$. https://doi.org/10.1177/014662167700100306

Shultz, J. M., Althouse, B. M., Baingana, F., Cooper, J. L., Espinola, M., Greene, M. C., ... Rechkemmer, A. (2016) Fear factor: The unseen perils of the Ebola outbreak. Bulletin of the Atomic Scientists, 72(5), 304-310. https://doi.org/10.1080/00963402.2016.1216515

Sekhon, Y. K. \& Szmigin, I. (2011). Acculturation and identity: insights from second-generation Indian Punjabis. Consumption Markets \& Culture, 14(1), 79-98. https://doi.org/10.1080/10253860903562171

Shevlin, M., McBride, O., Murphy, J., Miller-Gibson, J., Hartman, T., Levita, L., ... Bentall, R. (2020). Anxiety, depression, traumatic stress, and COVID-19 related anxiety in the UK general population during the COVID-19 pandemic. BJPsych Open, 6(6), e125. https://doi.org/10.1192/bjo.2020.109

Spinner-Halev, J., \& Theiss-Morse, E. (2003). National identity and self-esteem. Perspectives on Politics, 1(3), 515-532. https://doi.org/10.1017/S1537592703000379

Spitzer, R. L., Kroenke, K., Williams, J. B., \& Löwe, B. (2006). A brief measure for assessing generalized anxiety disorder: the GAD-7. Archives of Internal Medicine, 166(10), 1092-7. https://doi.org/10.1001/archinte.166.10.1092

Tajfel, H. \& Turner, J. C. (1986). The social identity theory of intergroup behaviour. In W. Austin \& S. Worchel (eds.), Psychology of Intergroup Relations (pp. 7-24). Chicago, IL: Nelson-Hall.

Torales, J., O'Higgins, M., Castaldelli-Maia, J., \& Ventriglio, A. (2020) The outbreak of COVID-19 coronavirus and its impact on global mental health. International Journal of Social Psychiatry, 66(4), 317-320. https://doi.org/10.1177/0020764020915212

Towers, S., Afzal, S., Bernal, G., Bliss, N., Brown, S., Espinoza, B., ... Castillo-Chaves, C. (2015). Mass media and the contagion of fear: the case of Ebola in America. PLoS One, 10, e0129179. https://doi.org/10.1371/journal.pone.0129179

UK Census (2011). UK population by ethnicity. https://www.ethnicity-factsfigures.service.gov.uk/uk-population-by-ethnicity

Vadher, K. \& Barrett, M. (2009). Boundaries of Britishness in British Indian and Pakistani young adults. Journal of Community and Applied Social Psychology, 19(6), 442-458. https://doi.org/10.1002/casp.1006

Wakefield, J. R. H., Bowe, M., Kellezi, B., McNamara, N., \& Stevenson, C. (2019). When groups help and when groups harm: Origins, developments, and future directions of the "Social Cure" perspective of group dynamics. Social and Personality Psychology Compass, 13, e12440. https://doi.org/10.1111/spc3.12440

Wang, C., Pan, R., Wan, X., Tan, Y., Xu, L., Ho, C. S., \& Ho, R. C. (2020). Immediate psychological responses and associated factors during the initial stage of the 2019 coronavirus disease (COVID-19) epidemic among the general population in China. International Journal of Environmental Research and Public Health, 17(5), 1729. https://doi.org/10.3390/ijerph17051729

Williams, D. R., Yu, Y., Jackson, J. S., \& Anderson, N. B. (1997). Racial differences in physical and mental health: socio-economic status, stress and discrimination. Journal of Health Psychology, 2(3), 335-351. https://doi.org/10.1177/135910539700200305

World Health Organization (2020). Statement on the second meeting of the International Health Regulations (2005) Emergency Committee regarding the outbreak of novel coronavirus (2019nCoV). https://www.who.int/news-room/detail/30-01-2020-statement-on-the-second-meeting-ofthe-international-health-regulations-(2005)-emergency-committee-regarding-the-outbreak-of-novelcoronavirus-(2019-ncov)

Xiong, J., Lipsitz, O., Nasri, F., Lui, L., Gill, H., Phan, L., ... McIntyre, R. S. (2020). Impact of COVID-19 pandemic on mental health in the general population: A systematic review. Journal of Affective Disorders, 277, 55-64. https://doi.org/10.1016/j.jad.2020.08.001 
Ysseldyk, R., Matheson, K., \& Anisman, H. (2010). Religiosity as identity: Toward an understanding of religion from a social identity perspective. Personality and Social Psychology Review, 14(1), 6071 https://doi.org/10.1177/1088868309349693

Yoo, H. C., \& Lee, R. M. (2005). Ethnic identity and approach-type coping as moderators of the racial discrimination/well-being relation in Asian Americans. Journal of Counseling Psychology, 52(4), 497-506. https://doi.org/10.1037/0022-0167.52.4.497 
Table 1. Means and standard deviations for the ethnic groups (British South Asian vs. British Black) for the key variables of interest

\begin{tabular}{|c|c|c|c|c|c|c|}
\hline & $\begin{array}{l}\text { British South Asian: } \\
\text { Indians and Pakistanis } \\
N=121\end{array}$ & & $\begin{array}{l}\text { British Black: } \\
\text { Black Africans and Black } \\
N=105\end{array}$ & aribbeans & Independent samples t-tests Cohen's ds & $95 \% C I$ \\
\hline Generalized anxiety & $\begin{array}{l}M \\
6.32\end{array}$ & $\begin{array}{c}S D \\
4.72\end{array}$ & $\begin{array}{l}M \\
4.62\end{array}$ & $\begin{array}{c}S D \\
4.33\end{array}$ & 0.4 & $0.48615,2.90470$ \\
\hline Fear of COVID-19 & $\begin{array}{l}M \\
26.67\end{array}$ & $\begin{array}{l}S D \\
8.10\end{array}$ & $\begin{array}{l}M \\
24.51\end{array}$ & $\begin{array}{l}S D \\
7.26\end{array}$ & 0.3 & $0.12579,4.08231$ \\
\hline Ethnic discrimination & $\begin{array}{l}M \\
2.13\end{array}$ & $\begin{array}{l}S D \\
0.76\end{array}$ & $\begin{array}{l}M \\
2.49\end{array}$ & $\begin{array}{l}S D \\
0.75\end{array}$ & 0.5 & $-5.6996,-0.16434$ \\
\hline Religious discrimination & $\begin{array}{l}M \\
1.77\end{array}$ & $\begin{array}{c}S D \\
0.69\end{array}$ & $\begin{array}{l}M \\
1.45\end{array}$ & $\begin{array}{c}S D \\
0.64\end{array}$ & 0.5 & $0.13753,0.49544$ \\
\hline Strength of ethnic identification & $\begin{array}{l}M \\
30.35\end{array}$ & $\begin{array}{l}S D \\
7.06\end{array}$ & $\begin{array}{l}M \\
32.60\end{array}$ & $\begin{array}{l}S D \\
6.32\end{array}$ & 0.3 & $\begin{array}{l}-0.02062,- \\
0.19070\end{array}$ \\
\hline $\begin{array}{l}\text { Strength of British national } \\
\text { identification }\end{array}$ & $\begin{array}{l}M \\
23.22 \\
\end{array}$ & $\begin{array}{l}S D \\
5.14 \\
\end{array}$ & $\begin{array}{l}M \\
20.37 \\
\end{array}$ & $\begin{array}{c}S D \\
4.91 \\
\end{array}$ & 0.6 & $1.57147,4.06343$ \\
\hline
\end{tabular}


Table 2. Stepwise regression model predicting the variance of depression

\begin{tabular}{|c|c|c|c|c|c|c|c|}
\hline & Depression & & & & & & \\
\hline Predictors & $R^{2}$ & $F$ & $p$ & $\beta$ & $t$ & $p$ & $95 \% C I$ \\
\hline Model 1 & 0.06 & 15.25 & $<0.001$ & & & & \\
\hline $\begin{array}{l}\text { Ethnic } \\
\text { discrimination }\end{array}$ & & & & 0.25 & 3.91 & $<0.001$ & $0.821,2.319$ \\
\hline Model 2 & 0.09 & 11.97 & $<0.001$ & & & & \\
\hline $\begin{array}{l}\text { Ethnic } \\
\text { discrimination }\end{array}$ & & & & 0.26 & 4.14 & $<0.001$ & $0.906,2.420$ \\
\hline Religiosity & & & & -0.18 & -2.86 & 0.005 & $-0.297,-0.128$ \\
\hline Model 3 & 0.19 & 17.40 & $<0.001$ & & & & \\
\hline $\begin{array}{l}\text { Ethnic } \\
\text { discrimination }\end{array}$ & & & & 0.20 & 3.15 & 0.002 & $0.542,1.888$ \\
\hline Religiosity & & & & -0.20 & -3.33 & 0.001 & $-0.312,-0.138$ \\
\hline Fear of COVID-19 & & & & 0.31 & 5.06 & $<0.001$ & $0.107, .0240$ \\
\hline Model 4 & 0.23 & 16.63 & $<0.001$ & & & & \\
\hline $\begin{array}{l}\text { Ethnic } \\
\text { discrimination }\end{array}$ & & & & 0.17 & 2.81 & 0.005 & $0.403,1.801$ \\
\hline Religiosity & & & & -.014 & -2.27 & 0.024 & $-0.271,-0.065$ \\
\hline Fear of COVID-19 & & & & 0.30 & 4.99 & $<0.001$ & $0.101,0.237$ \\
\hline Life satisfaction & & & & -0.21 & -3.43 & 0.001 & $-0.223,-0.085$ \\
\hline
\end{tabular}


Table 3: Stepwise regression model predicting the variance of generalized anxiety

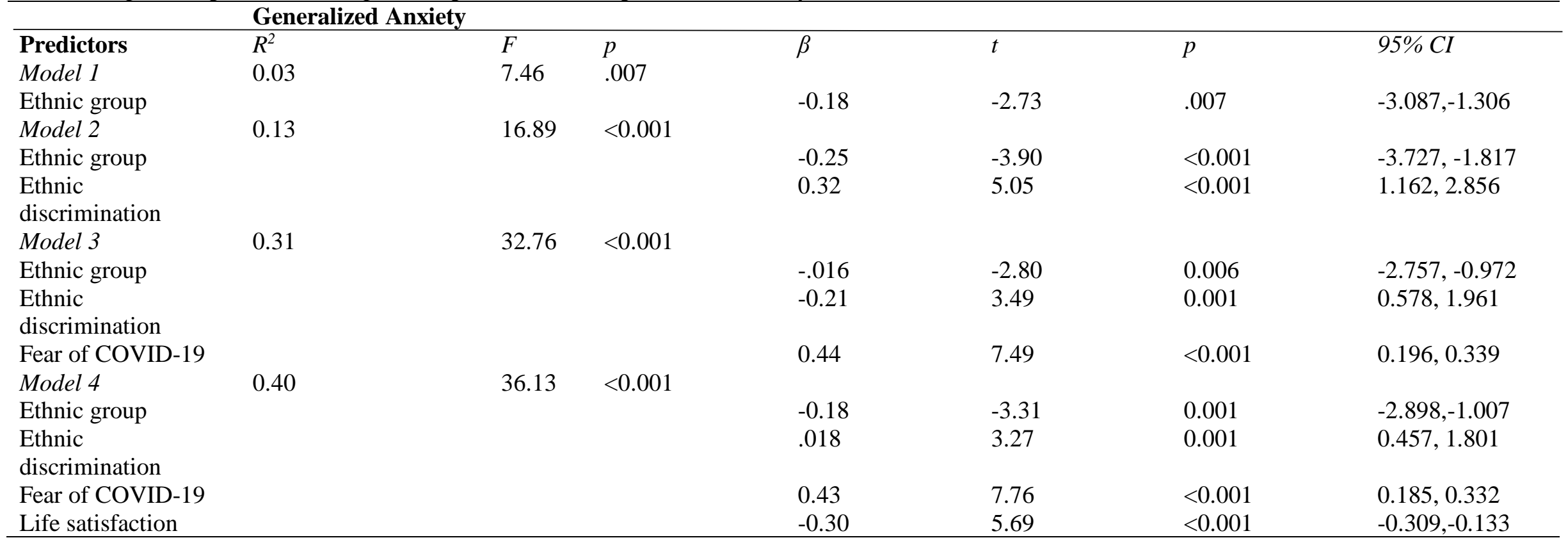




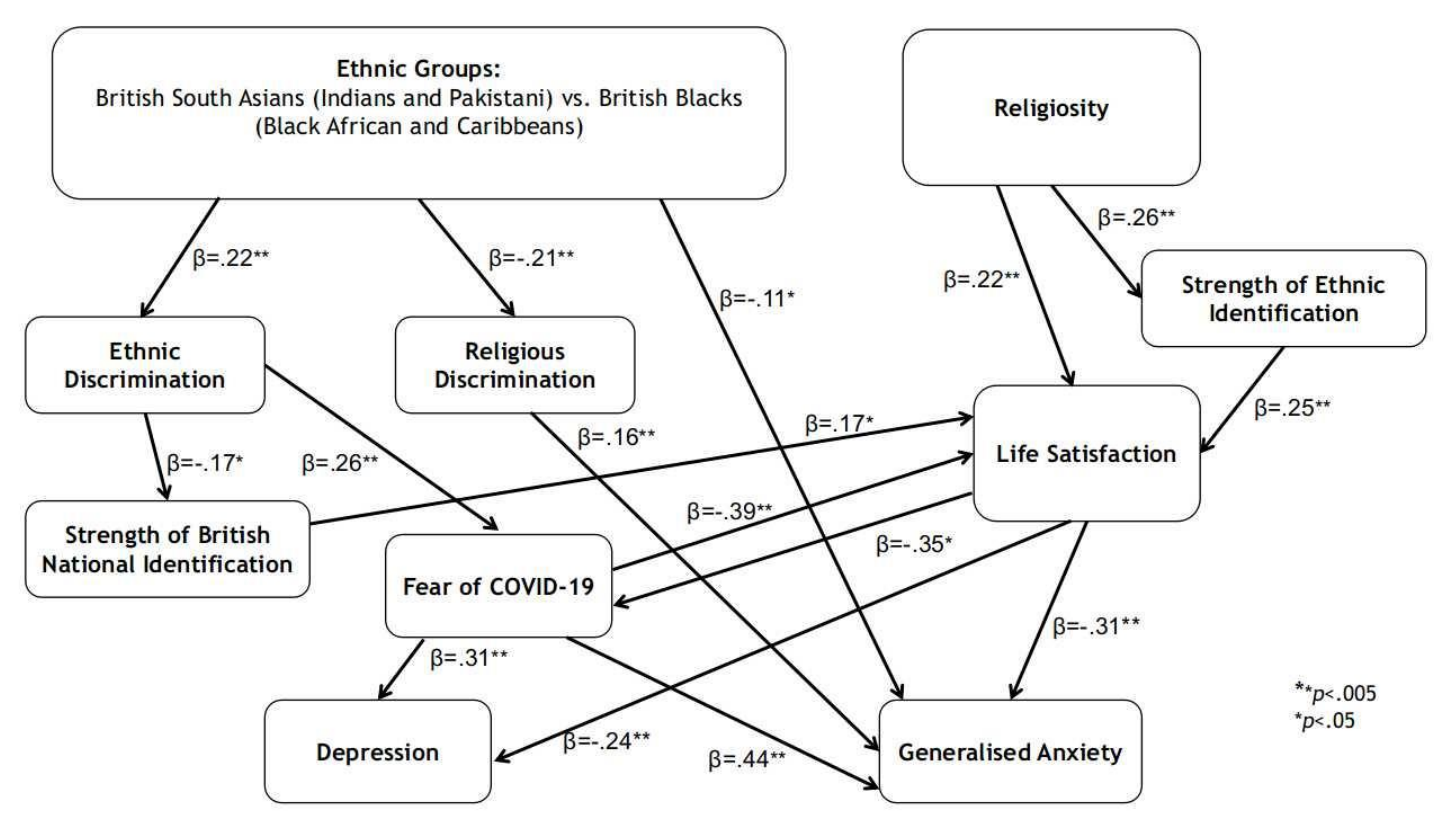

Figure 1. Structural equation model depicting the direct and indirect effects of ethnic groups and religiosity on depression and generalized anxiety through the mediators of ethnic discrimination, religious discrimination, strength of British national identification, strength of ethnic identification, fear of COVID-19 and life satisfaction 